\title{
Prediction of the weld pool geometry of TIG arc welding by using fuzzy logic controller
}

\author{
H. K. Narang ${ }^{1}$, U. P. Singh ${ }^{2}$, M. M. Mahapatra ${ }^{3}$ and P. K. Jha ${ }^{3}$ \\ ${ }^{1}$ Mechanical \& Industrial Engineering Department, Indian Institute of Technology, Roorkee, INDIA- 247667 \\ ${ }^{2}$ Mechanical \& Industrial Engineering Department, Indian Institute of Technology, Roorkee, INDIA- 247667 \\ ${ }^{3}$ Mechanical \& Industrial Engineering Department, Indian Institute of Technology, Roorkee, INDIA- 247667
}

\begin{abstract}
The present paper describes fuzzy logic simulation of tungsten inert gas welding (TIG) process to predict the weldment macrostructure zones' shape profile characteristics. The prediction of the weld pool geometry together with the shape of the heat affected zone (HAZ) was accomplished taking into account of TIG welding process parameters such as arc traverse speed, welding current and arc length. Structural steel plates of $8 \mathrm{~mm}$ thickness were used for the experiments. Full factorial design of experiment methodology was followed while selecting the input process parameters (control factors). These control factors were having three levels. The TIG weld pool geometry profiles' (boundaries of thermal cycle zones) like the weld bead reinforcement, penetration and heat affected zones were the responses from the experiment. A series of 27 experiments were carried out for collecting the data. The experimental data were then used for building a fuzzy logic model to predict the effects of control factors on the responses. A graphical mapping scheme was employed for the graphical representation of the macrostructure zones' shape profiles including that of HAZ. The model was also tested for a number of test cases to establish it's adequacy. The fuzzy logic modeling technique employed in the present investigation can be used for online prediction of the TIG weldment shape profiles. The methodology adopted in the present investigation indicated adequacy of fuzzy logic model for predicting the TIG weld-pool geometry and HAZ.
\end{abstract}

Keywords: TIG welding, weld width, weld penetration, depth of HAZ, Fuzzy-Logic Controller.

DOI: http://dx.doi.org/10.4314/ijest.v3i9.6

\section{Introduction}

During fusion arc welding, the weldment experiences thermal cycles that are characterized with very rapid heating and cooling. The fusion arc weldment is essentially a cast structure which undergoes rapid heating with temperature exceeding the melting point and followed with rapid solidification. The fusion weldment shape is the final characteristics of the heat convection and conduction in the liquid pool during the welding process. In addition to the formation of the weld, the weld thermal cycles also affect a portion of base material, beyond the weld zone, that is not melted but nevertheless metallurgically affected. This zone of the weldment is known as the heat affected zone (HAZ). The grain structures within the HAZ are varied and have different metallurgical properties to that of the weld. The weldment shape together with the shape of HAZ is the final output of the welding process and defines the overall integrity of the welded joint. Accurate prediction of the weld shape together with that of HAZ helps in avoiding costly and time consuming experiments. Moreover, it also improves the productivity of the welding process. The present paper discusses the prediction of TIG weldment shape profiles including that of HAZ by utilizing fuzzy logic modeling and full factorial experiments. Like most of the arc welding processes, the TIG process is non-linear and imbibed with a degree of uncertainties. However, the TIG welding process is also characterized with low heat input, less spatter and cleanness of the weld. The TIG welding uses non-consumable tungsten electrode and an inert gas such as argon for the shielding purpose.

Prediction of weld geometry has been a topic of great interest for the researchers as it is the result of entire weld thermal cycle. For predicting the weld geometry investigators have used statistical, soft computing and numerical approaches (Tarng and Yang, 
1998). Tarng et al. (1999) had used Taguchi methods and analysis of variance (ANOVA) for predicting the front width, back width, front height and back height of TIG welds. However, the prediction of heat affected zone was not attempted in this work (Tarng and Yang, 1998). Artificial neural network and fuzzy clustering was used by Tarng et al. (1999) to predict the front width, back width, front height and back height of TIG welds. However prediction of entire weld bead geometry including that of HAZ was not attempted (Tarng at al., 1999). Manufacturing procedures such machining, welding are non-linear in nature and often a very accurate numerical modeling considering a number of varying process parameters may not be feasible. Fuzzy logic modeling method is one of the artificial intelligence (AI) technique which has been in use for the modeling of manufacturing process such as welding and machining (Ali and Zhang, 1999). It is known that the fusion arc welding voltage signals are noisy associated with uncertainties due to disturbance associated with electrode tip, droplet formation, droplet detachment etc. (Bingül et al., 2000). Sensing the fusion arc position is hence associated with uncertainties.

Bingül et al. (2000) had developed a fuzzy controller for sensing and controlling the torch position of pulsed gas metal arc welding (P-GMAW). The weld penetration provides important information regarding the formation of back bead width (Gao and $\mathrm{Wu}, 2002)$. Moreover the weld penetration defines the overall joint integrity of a TIG weld. The weld pool image provides most of the information related to weld penetration and bead geometry. Gaoand Wu had predicted the back width of TIG welds using the images of the weldment pool and artificial neural networks (ANN) (Gao and $\mathrm{Wu}, 2002$ ). The fusion weld pool has been the ultimate interest of welding researchers as it is the final result of the welding and defines the overall weld structural integrity. For sensing the weld pool researchers in past have utilized many sensing devices such as charge coupled device (CCD) camera and predicted the weld characteristics (Wu et al., 2003). Most of the work related to fusion arc weld bead modelling has been limited to the prediction of bead characteristics such as bead height, bead widths and depth of penetration. However, one of the pioneering research contribution related to prediction of complete weld bead geometry was presented by Kim et al. (2005). In this work, ANN and multiple regression models were used for GMAW bead geometry and online graphical representation of the complete bead geometry was made (Kim et al., 2005). An intelligent optical sensor to detect the defects in GMAW welds was developed by Naso et al. (2005) using fuzzy logic system. The sensor developed (Naso et al., 2005), measured the radiations emitted by the plasma which surrounded the arc and analysed the data using a Kalman filter to reduce the noise. The sensed data was also used with an intelligent fuzzy system to detect the positions of defects along the weld line (Naso et al., 2005).

The performance of high-frequency arc welder was evaluated with a fuzzy logic controller and state space averaging based sliding control by İskender and Karaarslan (2005). It was observed in their work that the fuzzy logic controller was more robust (İskender and Karaarslan, 2005). An unsupervised fuzzy clustering algorithm was employed by Kim et al. (2006) to predict the GMAW bead geometry. In their work the GMAW bead height, bead width, weld penetration and weld cross sectional area were predicted accurately by using the fuzzy clustering algorithm (Kim et al., 2006). An ANN model was developed by Wu et al. to predict the front side geometrical parameters and back side weld width of gas tungsten arc welds (Wu et al., 2006). It was suggested that since the back side weld width described the weld penetration, the information of front side weld width could be used for controlling the overall weld features (Wu et al., 2006). TIG weld characteristics are strongly influenced by the weld pool geometry and as such various methodologies have been investigated by the researchers for its prediction. Esmeet al. (2009) developed a mathematical model to predict the TIG weld bead characteristics (Esme et al., 2009). In their work the weld bead was characterized with its quality features such as upper bead width, lower bead width, penetration depth etc and mathematically related to the input parameters such as welding speed, welding current, shielding gas flow rate and arc gap distance (Esme et al., 2009). Optimization and prediction of weld bead geometry of GMAW welds was done by Kolahan and Heidari (2011) by using curvilinear regression models. The weld beads were also optimized using a Simulated Annealing (SA) optimization algorithm (Kolahan and Heidari, 2011). Podržaj and Simon (2011) developed a fuzzy logic controller for detecting expulsion of resistance spot welds during the welding and for stopping the welding process to avoid unnecessary electrode degradation.

As different to conventional approach of modeling the welding process, the fuzzy logic modeling technique provides a good solution. Although significant achievements have been made in this area, direct, accurate, reliable and real-time techniques for predicting the TIG weldment shape together with the HAZ is more promising due to the specific limitations associated with each individual method. Most of the research work related to arc weld pool geometry involves prediction of maximum bead reinforcement and width. Very rarely a complete prediction of weldment shape profile including that of HAZ is observed in the contemporary literature. This investigation presents a basis to predict the effect of a variety of process parameters (welding speed, welding current, and arc length) on the weld pool geometry (heat affected zone, bead width, depth of penetration and area of penetration). Test cases were also probed to verify the adequacy of the fuzzy model developed for predicting the weldment characteristics and shape profiles. A general structure of fuzzy interference system is shown in Fig. 1. 


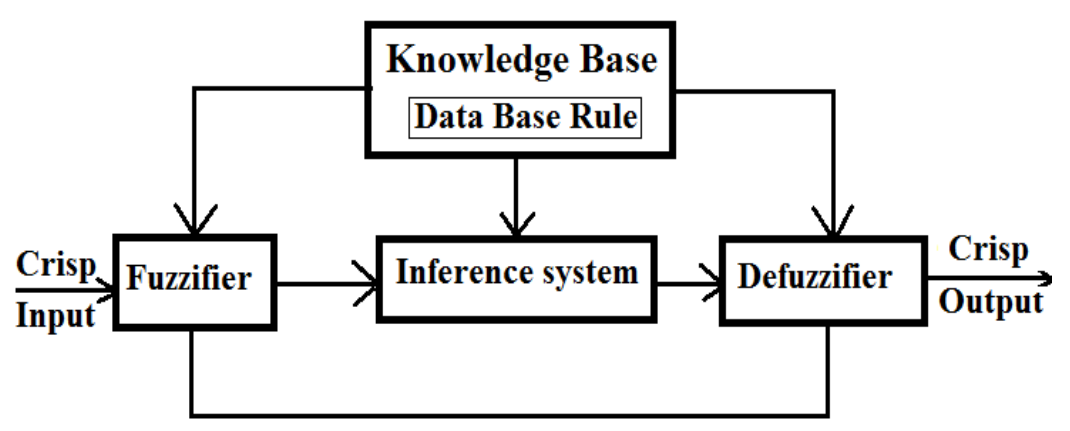

Fig. 1. General Structure of fuzzy inference system

\section{Experimental Details}

TIG welding with direct current straight polarity (DCSP) and integrated with arc image magnifying system was used in experiments. The experimental setup of TIG welding integrated with linear variable displacement transformer (LVDT) is shown in Fig.2. The LVDT was used during experiment to set the gap between the electrode tip and work piece. TIG welding with DCSP was used in the experiment as it concentrates the heat in the joint area. In direct current electrode negative polarity (sometimes called as straight polarity) the work would be connected to the positive terminal. Trial runs were initially conducted for the beadon-plates to set the levels of three welding input process parameters such as welding current, traverse speed and arc length. The trials experiment runs were conducted to select the range of welding input parameter such that no observable defects like undercutting and porosity occurred. The chemical composition of structural steel is given in Table 1.

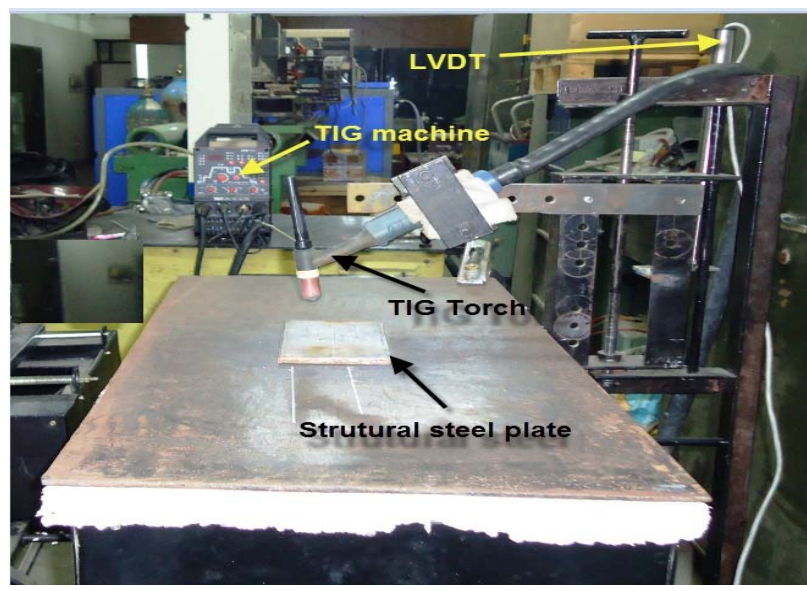

Fig. 2 Experimental set-up of TIG welding

Table 1 Chemical composition of structural steel

\begin{tabular}{|c|c|c|c|c|c|c|c|}
\hline C \% & Si \% & Mn \% & P \% & S \% & Ni \% & Cr \% & Fe \% \\
\hline 0.16 & 0.178 & 0.45 & 0.18 & 0.07 & 0.13 & 0.016 & 98.84 \\
\hline
\end{tabular}

Proper selection of welding current, welding speed and argon gas flow rates plays important part in controlling the TIG weld quality. The 27 test experiments of bead on plates were conducted to obtain test data for building the fuzzy model. The length, width and thickness of mild steel plates used in the experiments were 180,65, and $8 \mathrm{~mm}$ respectively. Before conducting the final experiments, structural steel plates were cleaned properly to avoid the unwanted scaling. During the welding, gas flow rate and diameter of TIG electrode were kept constant. The polished and etched TIG weldment cross sections produced using different process parameters are shown in Fig. 3. 

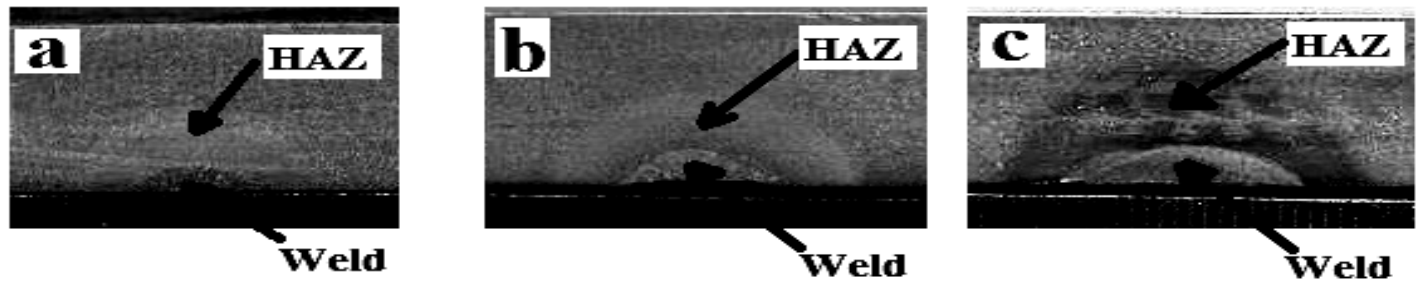

Figs.3. TIG welds polished and etched showing the weldments and HAZ with process parameters such as current, arc length and traverse speeds: (a) $55 \mathrm{~A}, 2 \mathrm{~mm}, 15 \mathrm{~mm} / \mathrm{sec}$; (b) $75 \mathrm{~A}, 2.5 \mathrm{~mm} 30 \mathrm{~mm} / \mathrm{sec}$; and (c) $95 \mathrm{~A}, 3 \mathrm{~mm}, 45 \mathrm{~mm} / \mathrm{sec}$ respectively

\section{Bead geometrical characteristics mapping}

The experience of TIG indicates the repeatability of the process which facilitates modeling the weld pool geometry. The TIG geometry can be cross sectioned as shown schematically in Fig. 4 and the various zones of it can be measured. From Fig. 4, it can be observed that the HAZ width (HW) is the distance AB and DE. Similarly BD, CF and FG can be measured for representing bead width (BW), penetration depth (P) and depth of HAZ (DHAZ) respectively. Hence the fusion zone can be graphically represented by curve fitting of the points B-C-D-F-B (Fig. 4). Similarly, from the Fig. 4 it can be stated that the HAZ can be graphically represented by the curve fitting of the points A-B-F-D-E-G-A. Weld samples were cut from the test pieces and polished by silicon carbide paper with different grades and etched with Nital solution (5-10\%, Nitric Acid with water) and then metallographic test was performed to obtain detailed measured features of complete weld pool geometries (bead width, depth of penetration, width of HAZ and depth of HAZ) as shown in Fig. 4.

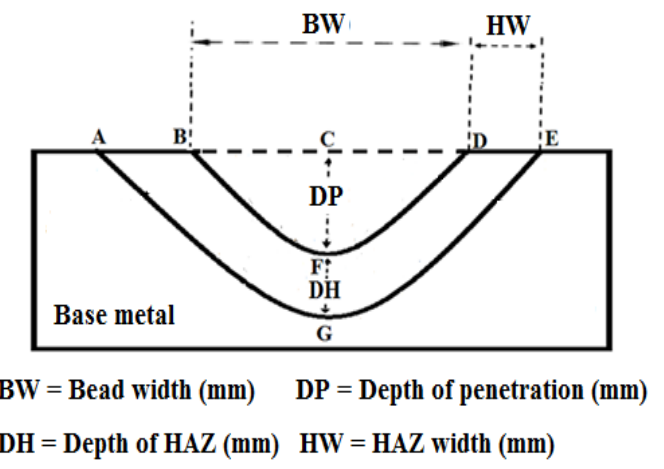

Fig. 4. Schematic of TIG weld pool geometry shape profile showing bead width (BW), HAZ width (HW), depth of penetration (DP) and depth of HAZ (DH)

The welds have shown unpredictable and abnormal upper surface depression. The upper surface depression was random in nature and did not occur for all the 27 experiments. The occurrence of upper surface depression is represented by dotted line as shown in Fig. 4. The dimensions of various zones can be utilized in graphical software for representing the complete weldment shape profile based on the experimental data as shown Table 2 by curve fitting. The fuzzy model was derived to study the suitability of the fuzzy relations for predicting the weldment shape profile characteristics of test cases. The full factorial design method was employed to have enough experimental data for building the fuzzy model to predict the weld pool geometry.

\section{Fuzzy model construction}

The TIG welding is dependent on several parameters which can be varying over a wide range. Initially, feasibility study was carried out to predict the overall acceptability of welds. Fuzzy logic is a highly flexible and non linear modelling technique with an ability to learn the relationship between inputs variables and output features. The most successful applications of fuzzy set theory is observed in modeling the experimental data involving certain uncertainties between the relationships of input process variables and responses (Bandler and Kohout, 1988). Its major features are the use of linguistic variables rather than numerical variables. Linguistic variables are defined as the variables whose values are sentences in natural language (such as low, medium and high) and can be represented by fuzzy sets. Fuzzy sets are characterized by fuzzification, membership functions, a fuzzy rule, an inference system and a defuzzification inference. The structure of three inputs, four outputs fuzzy logic controller developed for this present research is shown in Fig. 5. The inputs values to the model were given in linguistic form and after fuzzification, the outputs were obtained in crisp form. 
Table 2. The experimental data of TIG arc weld experiment

\begin{tabular}{|c|c|c|c|c|c|c|c|}
\hline S.N & $\begin{array}{l}\text { Current } \\
\text { (A) }\end{array}$ & $\begin{array}{l}\text { Arc length } \\
\quad(\mathrm{mm})\end{array}$ & $\begin{array}{c}\text { Welding Speed } \\
(\mathrm{mm} / \mathrm{s})\end{array}$ & $\begin{array}{l}\text { Bead width } \\
\quad(\mathrm{mm})\end{array}$ & $\begin{array}{c}\text { Depth of } \\
\text { penetration } \\
(\mathrm{mm})\end{array}$ & $\begin{array}{c}\text { Depth of } \\
\text { HAZ } \\
(\mathrm{mm})\end{array}$ & $\begin{array}{c}\text { Width of } \\
\text { HAZ } \\
(\mathrm{mm})\end{array}$ \\
\hline 1 & 55 & 2 & 15 & 5.46 & 1.59 & 1.73 & 1.83 \\
\hline 2 & 55 & 2 & 30 & 4.71 & 1.25 & 1.19 & 1.35 \\
\hline 3 & 55 & 2 & 45 & 4.16 & 1.04 & 1.02 & 1.13 \\
\hline 4 & 55 & 2.5 & 15 & 5.77 & 1.76 & 1.94 & 2.20 \\
\hline 5 & 55 & 2.5 & 30 & 4.93 & 1.38 & 1.33 & 1.63 \\
\hline 6 & 55 & 2.5 & 45 & 4.46 & 1.18 & 1.16 & 1.33 \\
\hline 7 & 55 & 3 & 15 & 6.09 & 1.91 & 2.13 & 2.45 \\
\hline 8 & 55 & 3 & 30 & 5.03 & 1.42 & 1.51 & 1.84 \\
\hline 9 & 55 & 3 & 45 & 4.55 & 1.23 & 1.23 & 1.46 \\
\hline 10 & 75 & 2 & 15 & 6.12 & 1.99 & 2.48 & 2.25 \\
\hline 11 & 75 & 2 & 30 & 5.13 & 1.39 & 1.46 & 1.72 \\
\hline 12 & 75 & 2 & 45 & 4.59 & 1.16 & 1.22 & 1.39 \\
\hline 13 & 75 & 2.5 & 15 & 6.59 & 2.06 & 2.65 & 2.41 \\
\hline 14 & 75 & 2.5 & 30 & 5.26 & 1.50 & 1.65 & 1.89 \\
\hline 15 & 75 & 2.5 & 45 & 4.85 & 1.32 & 1.34 & 1.57 \\
\hline 16 & 75 & 3 & 15 & 7.07 & 2.18 & 2.72 & 2.79 \\
\hline 17 & 75 & 3 & 30 & 5.45 & 1.65 & 1.86 & 2.02 \\
\hline 18 & 75 & 3 & 45 & 5.16 & 1.45 & 1.58 & 1.79 \\
\hline 19 & 95 & 2 & 15 & 6.65 & 2.17 & 3.04 & 2.71 \\
\hline 20 & 95 & 2 & 30 & 5.38 & 1.51 & 1.81 & 1.94 \\
\hline 21 & 95 & 2 & 45 & 4.75 & 1.23 & 1.49 & 1.52 \\
\hline 22 & 95 & 2.5 & 15 & 7.19 & 2.23 & 3.32 & 2.89 \\
\hline 23 & 95 & 2.5 & 30 & 6.16 & 1.63 & 1.97 & 2.15 \\
\hline 24 & 95 & 2.5 & 45 & 5.2 & 1.32 & 1.56 & 1.75 \\
\hline 25 & 95 & 3 & 15 & 7.64 & 2.51 & 3.21 & 3.15 \\
\hline 26 & 95 & 3 & 30 & 6.31 & 1.74 & 2.15 & 2.70 \\
\hline 27 & 95 & 3 & 45 & 5.11 & 1.41 & 1.74 & 2.16 \\
\hline
\end{tabular}

The fuzzy rules are expressed in the form of fuzzy conditional statements $R_{i}$ of the type $\mathrm{R}_{\mathrm{i}}$ : if $\mathrm{x}$ is small $\mathrm{y}$ is large THEN $\mathrm{z}$ is large

Where $\mathrm{x}$ and $\mathrm{y}$ are fuzzy variables, and small and large are labels of fuzzy set. If there are $\mathrm{i}=1$ to $\mathrm{n}$ rules, the rule set is represented by union of these rules

$R=R_{1}$ else $R_{2}$ else $\ldots \ldots . . . R_{n}$

INPUTS

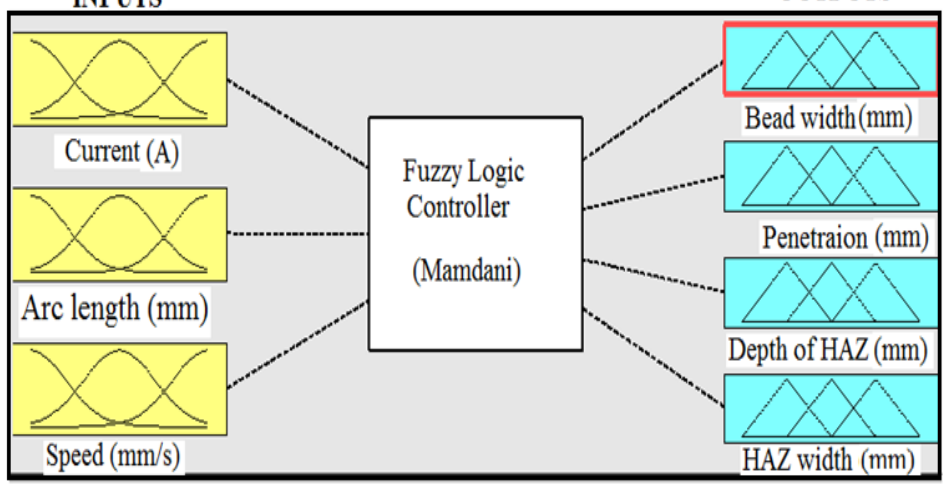

Fig. 5. Input-output parameters of Fuzzy logic control model 
A fuzzy logic controller is based on a collection of control rules. The execution of these rules is governed by the compositional rule. In this study the triangular membership functions were used for the input process parameters such as arc length, welding current and welding traverse speed to predict the TIG weldment shape profiles. The membership function for each inputs and outputs variables were divided into three levels (low, medium and high).The fuzzy logic controller was Mamdani type and contained a rule base. This base comprised of groups of rules and each output was defined by twenty seven rules. Hence total numbers of rules for the four outputs such as bead width, penetration depth, depth of HAZ and HAZ width were 108. The rules based on knowledge to predict the weldment shape profile characteristics of TIG bead-on-plates is given in Table 3 .

Table 3 Fuzzy rule for weldment characteristics of TIG welding

1. IF current is Low AND Voltage is Low AND Speed is Low THEN Bead width will be Medium

2. IF current is Low AND Voltage is Low AND Speed is Medium THEN Bead width will be Low

3. IF current is Low AND Voltage is Low AND Speed is High THEN Bead width will be Low

06. IF current is high AND voltage is High AND speed is Low THEN HAZ width will be High

107. IF current is high AND voltage is High AND speed is Medium THEN HAZ width will be Medium

108. IF current is high AND voltage is High AND speed is High THEN HAZ width will be Low

The fuzzy input and outputs are linguistically divided into three levels such as low (L), medium (M) and high (H) which is shown in Fig. 6 and Fig. 7 respectively. The model provides an excellent capability to predict the weldment shape profile in terms of bead width, penetration, depth of HAZ and width of HAZ.

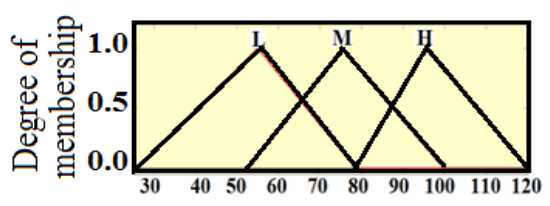

(a) Current (A)

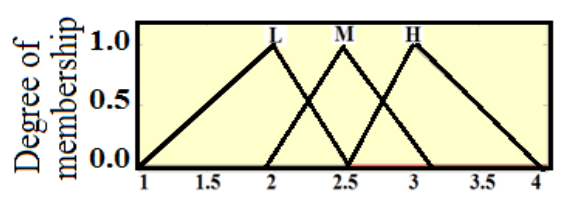

(b) Voltage (V)

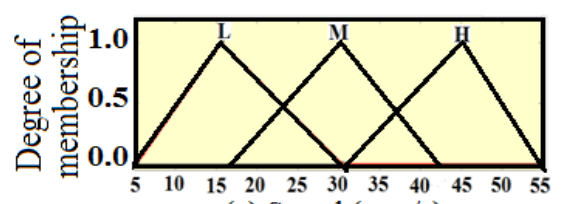

(c) Speed $(\mathrm{mm} / \mathrm{s})$

Fig.6. Membership functions for inputs process parameters: (a) Welding current; (b) Arc length; \& (c) Traverse speed of TIG weldment

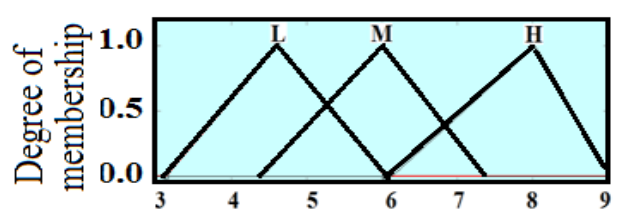

(a) Bead width, BW, (mm)

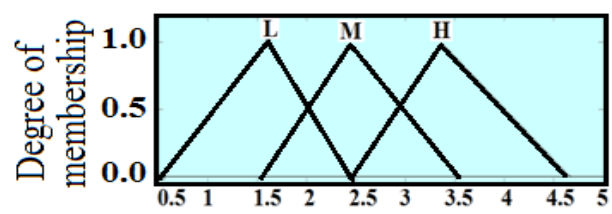

(c) Depth of HAZ, DH, (mm)

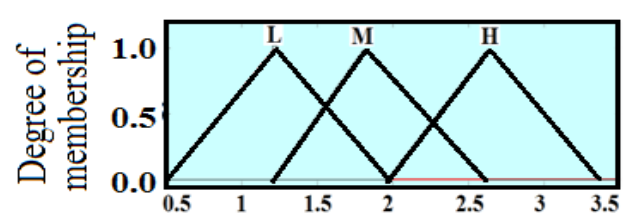

(b) Penetration, $\mathrm{P},(\mathrm{mm})$

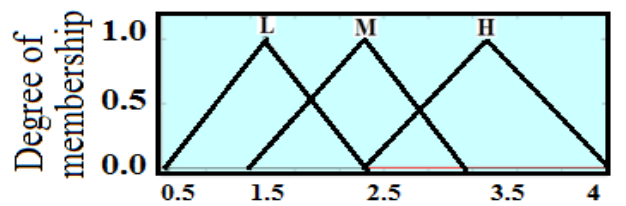

(d) HAZ Width, HW, (mm)

Fig.7. Membership functions for outputs: (a) Bead width (BW); (b) Depth of penetration (DP) ; (c) Depth of HAZ (DH); and (d) HAZ width (HW) of TIG weldment 


\section{Simulation of Fuzzy Logic Model}

In this study, the fuzzy model has been developed based on 27 experiments of TIG process parameters. The fuzzy model was simulated for test cases which has been done within the range of the fuzzy set. The experiments was conducted for the three levels of process parameters such as current $(55,75 \& 95$ Ampere), arc length $(2,2.5 \& 3 \mathrm{~mm})$ and welding speed $(15,30 \& 45$ $\mathrm{mm} / \mathrm{sec}$ ).The purpose of the simulation was to minimize the error of outputs for test case experiments. A MATLAB Simulink model was developed to predict the TIG weldment shape profiles. To confirm the adequacy of fuzzy logic model, test case inputs were used to predict the outputs from the model. The measured and predicted values of TIG weldments characteristics are given in Table 4 and the percentage error of fuzzy modeling analysis is presented in Table 5 for the test cases. The maximum percentage of error for prediction (for HAZ width) was observed to be 21.91 .

Table 4. Measured and predicted weldment dimensions for test cases

\begin{tabular}{|c|c|c|c|c|c|c|c|c|}
\hline S. & Measured & Predicted & Measured & Predicted & Measured & Predicted & Measured & Predicted \\
N & BW (mm) & BW (mm) & DP (mm) & DP (mm) & DH (mm) & DH (mm) & HW (mm) & HW (mm) \\
\hline 1 & 5.76 & 5.25 & 1.72 & 1.58 & 1.99 & 1.74 & 2.15 & 1.87 \\
\hline 2 & 5.17 & 5.05 & 1.69 & 1.60 & 2.05 & 1.79 & 2.01 & 1.88 \\
\hline 3 & 6.11 & 5.47 & 1.79 & 1.56 & 1.89 & 1.68 & 2.17 & 1.85 \\
\hline 4 & 6.44 & 5.70 & 1.84 & 1.62 & 2.09 & 1.81 & 2.50 & 2.05 \\
\hline 5 & 5.70 & 5.31 & 1.70 & 1.48 & 1.95 & 1.63 & 2.18 & 1.84 \\
\hline 6 & 5.16 & 5.13 & 1.62 & 1.52 & 1.82 & 1.62 & 2.09 & 1.98 \\
\hline 7 & 6.24 & 5.54 & 1.72 & 1.53 & 2.09 & 1.76 & 2.37 & 2.01 \\
\hline 8 & 6.31 & 5.51 & 1.71 & 1.56 & 2.26 & 1.91 & 2.31 & 2.03 \\
\hline 9 & 6.46 & 5.68 & 2.22 & 1.94 & 2.97 & 2.62 & 2.71 & 2.28 \\
\hline
\end{tabular}

Table 5. Percentage of error for test cases from Fuzzy logic simulation model

\begin{tabular}{|c|c|c|c|c|c|c|c|}
\hline S.N & $\begin{array}{c}\text { Current } \\
(\mathrm{A})\end{array}$ & $\begin{array}{c}\text { Arc } \\
\text { Length } \\
(\mathrm{mm})\end{array}$ & $\begin{array}{c}\text { Speed } \\
(\mathrm{mm} / \mathrm{s})\end{array}$ & $\begin{array}{c}\text { \% Error of } \\
\text { BW } \\
\text { prediction }\end{array}$ & $\begin{array}{c}\text { \% Error of } \\
\text { DP } \\
\text { prediction }\end{array}$ & $\begin{array}{c}\text { \%Error of } \\
\text { DH } \\
\text { prediction }\end{array}$ & $\begin{array}{c}\text { \%Error of } \\
\text { HW } \\
\text { prediction }\end{array}$ \\
\hline 1 & 55 & 1.8 & 15 & 9.78 & 8.63 & 14.46 & 14.54 \\
\hline 2 & 60 & 1.5 & 15 & 2.44 & 5.21 & 14.45 & 6.95 \\
\hline 3 & 65 & 2.3 & 23 & 11.66 & 14.96 & 12.62 & 17.30 \\
\hline 4 & 75 & 2.7 & 28 & 13.10 & 13.27 & 14.92 & 21.91 \\
\hline 5 & 75 & 2.7 & 35 & 7.46 & 14.74 & 19.91 & 18.42 \\
\hline 6 & 65 & 3.2 & 35 & 0.56 & 6.15 & 12.27 & 5.51 \\
\hline 7 & 85 & 2.7 & 35 & 12.69 & 12.67 & 18.71 & 17.74 \\
\hline 8 & 95 & 1.8 & 27 & 14.44 & 9.85 & 18.61 & 13.56 \\
\hline 9 & 85 & 1.5 & 15 & 13.56 & 14.38 & 13.37 & 18.92 \\
\hline
\end{tabular}

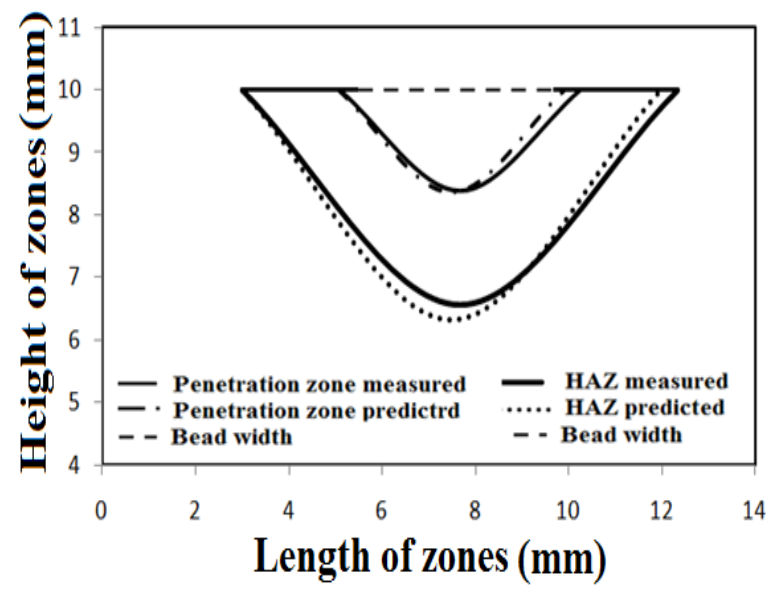

Fig. 8. TIG weldment shape profile of test case NO.2 (Table 4) 
The experimentally observed and predicted values of fuzzy outputs were used for graphical representation of the weldment cross-section shape profiles. The results from fuzzy logic simulation indicated that the predicted values and experimental values closely agreed. In some cases the predicted values and experimental values are observed to be little deviated. That might be due to some experimental error. The maximum deviation is shown in case of HAZ since it is very difficult to measure the exact HAZ boundary. The graphical representation of weld pool geometry (experimental and predicted) of TIG bead-on-plate for two test cases (No.2 \&6 Table 4) are presented in Figs 8\&9. It can be observed that there is good agreement between the experimental and predicted values of shape profile characteristics.

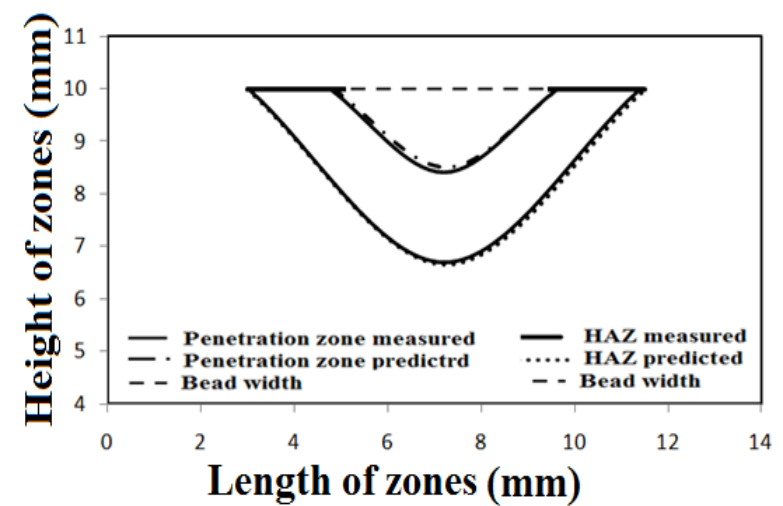

Fig.9. TIG weldment shape profile of test case No.6 (Table 4)

\section{Conclusions}

In this investigation TIG weldment shape profiles have been successfully predicted for the full factorial design of experimental data and for the test cases. The fuzzy clustering technique used was found to be adequate for establishing the relationship between the input process parameters and the outputs. The TIG weld pool geometry can be divided into a number of equal segments; corresponding weld deposit bead width, penetration depths and depths of HAZ at these segments can be used for effective graphical representation of the weldment geometry shape. A mapping technique was used which considered the depth of HAZ and width of HAZ such that, together with the weld bead geometry, the entire weldment shape profile could be graphically presented. The developed fuzzy logic model was also tested for a number of test cases, the input data of which was not used in the building of the fuzzy logic model. The prediction capability of the model for the test cases were evaluated and found to be adequate (within an accuracy limit of 22\%) for the graphical presentation of the TIG weldment shape profiles including that of the HAZ.

\section{References}

Ali Y.M. and Zhang L.C., 1999. Surface roughness prediction of ground components using a fuzzy logic approach, Journal of Materials Processing Technology, Vol. 89-90, pp. 561-568.

Bandler W. and Kohout L. J., 1988. Special properties, closures and interiors of crisp and fuzzy relations, Fuzzy sets and Systems, Vol. 26, No. 3, pp. 317-332.

Bingül Z., Cook G. E. and Strauss A. M., 2000. Application of fuzzy logic to spatial thermal control in fusion welding, IEEE transactions on industry applications, Vol. 36, No. 6, pp. 1523-1530.

Esme U., Kokangul A., Bayramoglu and Geren M. N., 2009. Mathematical modeling for prediction and optimization of TIG welding pool geometry, Journal of Metalurgija, Vol. 48, No. 2, pp. 109-112.

Gao J. and Wu C., 2002. Extracting weld penetration information in tungsten- inert gas welding, Proceedings of IMechE Part B: Journal of Engineering Manufacture, Vol. 216, pp. 207-214.

Iskender I. and Karaarslan A., 2005. On the comparison of fuzzy logic and state space averaging based sliding control methods applied on an arc welding machine, World Academy of Science, Engineering and Technology, Vol. 8, pp. 100-105.

Kim I.S., Son J.S., Park C.E., Kim. I.J. and Kim H., 2005. An investigation into an intelligent system for predicting bead geometry in GMA welding process. Journal of Materials Processing Technology, Vol. 159, No. 1, pp. 113-118.

Kim, I. S., Son, J. S., Kim, H. H., Kim, I. J., and Kang, B. Y., 2006. A study on fuzzy logic theory to predict the process parameters in GMA welding process, Materials Science Forum, Vol. 505-507, pp. 541-546.

Kolahan F. and Heidari M., 2011. A new approach for predicting and optimizing weld bead geometry in GMAW, International Journal of Aerospace and Mechanical Engineering, Vol. 5, No. 2, pp. 138-142.

Naso D., Turchiano B. and Pantaleo P., 2005. A fuzzy-logic based optical sensor for online weld defect-detection, IEEE Transactions on Industrial Informatics, Vol. 4, No. 1, pp. 259-273. 
Podrzaj P. and Simon S., 2011. Resistance spot welding control based on fuzzy logic, International Journal of Advanced Manufacturing Technology, Vol. 52, pp. 959-967.

Tarng Y. S. and Yang W. H., 1998. Optimization of weld bead geometry in gas tungsten arc welding. International Journal of advanced Manufacturing Technology, Vol. 14, pp. 549-554.

Tarng Y. S., Tsai H. L. and Yeh. S. S., 1999. Modeling, optimization and classification of weld quality in tungsten inert gas welding. Journal of Machine Tools and Manufacture, Vol. 39, pp. 1427-1438

Wu C. S., Gao J. Q., Liu X. F. and Zhao Y. H., 2003. Vision-based measurement of weld pool geometry In constant-current gas tungsten arc welding, Proceedings of IMechE Part B: Journal of Engineering Manufacture, Vol. 217, pp. 879-882.

Wu C. S., Gao, J. Q. and Zhao Y. H., 2006. A neural network for weld penetration control in gas tungsten arc welding, J. of Acta Metall. Sin. (Engl. Lett.), Vol. 19, No. 1, pp. 27-33.

\section{Biographical notes}

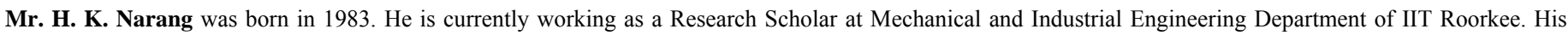
research topic is modeling the effects of open and covered arc welding process parameters on weldment characteristics and distortion.

Mr. U. P. Singh has completed post graduate studies in Mechanical and Industrial Engineering Department of IIT Roorkee. His research topic was related to full factorial design of experiment based TIG welding for optimization of the welding conditions.

Dr. M. M. Mahapatra was born in 1970. He is working as Assistant Professor of Mechanical and Industrial Engineering Department at Indian Institute of Technology, Roorkee. His current research interests include plate forming by line heating, welding deformation, welding residual stress analysis and designing of welded structures.

Dr. P. K. Jha was born in 1971. He is an Assistant Professor of IIT Roorkee, Dept. of Mechanical and Industrial Engineering. His area of expertise include process modeling, metal casting. He has, to his credit about 25 research publications in journals and conferences.

Received December 2011

Accepted June 2012

Final acceptance in revised form August 2012 